\title{
Effects of bone marrow-derived mesenchymal stem cells on the autophagic activity of alveolar macrophages in a rat model of silicosis
}

\author{
HUI-XING ZHU ${ }^{1}$, JUN-LING GAO ${ }^{1}$, MAN-MAN ZHAO ${ }^{1}$, RAN LI $^{1}$, YAN-XIA TIAN ${ }^{1}$, \\ $\mathrm{XIN}$ WANG $^{1}$, JUAN ZHANG ${ }^{1}$, JU-XIANG YUAN ${ }^{1}$ and JIAN-ZHONG CUI ${ }^{2}$ \\ ${ }^{1}$ Department of Histology and Embryology, School of Basic Medical Sciences, Hebei United University; \\ ${ }^{2}$ Department of Neurosurgery, Tangshan Workers' Hospital, Tangshan, Hebei 063000, P.R. China
}

Received August 6, 2014; Accepted April 27, 2015

DOI: 10.3892/etm.2016.3200

\begin{abstract}
The aim of the present study was to evaluate the effects of bone marrow-derived mesenchymal stem cells (BMSCs) on the expression of the autophagy-associated proteins, microtubule-associated protein light chain 3 (LC-3) and autophagy-related gene Beclin-1 (Beclin-1), in alveolar macrophages (AMs) in a rat model of silicosis. Furthermore, the study investigated the molecular mechanisms underlying the effects of BMSC treatment. A population of 60 adult female Sprague-Dawley (SD) rats were allocated at random into three groups, namely the control, model and BMSC treatment groups ( $n=20$ per group). BMSCs were isolated from five male SD rats (age, 6-8 weeks) and cultured in vitro. The silicosis model was established using a single $1.0-\mathrm{ml}$ infusion of silicon dioxide suspension administered via non-exposed tracheal intubation. Rats in the BMSC treatment group received a $1.0-\mathrm{ml}$ transplantation of BMSCs $(1 \times 106 / \mathrm{ml})$. The rats were sacrificed on days 1, 7, 14 and 28 after modeling, and AMs were extracted from the rats using bronchoalveolar lavage. Third-generation BMSCs were identified using flow cytometry with fluorescein isothiocyanate staining, and the morphological characteristics of the AMs were observed using hematoxylin and eosin staining. The expression levels of LC-3 and Beclin-1 were determined using immunocytochemistry
\end{abstract}

Correspondence to: Dr Jun-Ling Gao, Department of Histology and Embryology, School of Basic Medical Sciences, Hebei United University, 57 South Jianshe Road, Tangshan, Hebei 063000, P.R. China

E-mail: junlinggao1960@126.com

Abbreviations: BMSCs, bone marrow-derived mesenchymal stem cells; AM, alveolar macrophage; LC-3, microtubule-associated protein light chain 3; Beclin-1, autophagy-related gene Beclin-1; SD, Sprague-Dawley; BAL, bronchoalveolar lavage; PBS, phosphate-buffered saline; DMEM, Dulbecco's modified Eagle's medium; HE, hematoxylin and eosin

Key words: bone marrow-derived mesenchymal stem cells, silicosis, alveolar macrophages, autophagy sand western blot analysis. The expression levels of LC-3 and Beclin-1 were found to be increased at all the time points in the model group. LC-3 and Beclin-1 levels began to increase at day 1, peaked at day 14 and decreased after day 28; however, the levels remained elevated compared with the basal expression levels. The AMs of the BMSC treatment group exhibited significantly alleviated pathological symptoms compared with the model group AMs, as indicated by significantly decreased expression levels of LC-3 and Beclin-1 at each time point. Therefore, the results indicated that autophagy was promoted in the AMs of the silicosis model rats. Furthermore, treatment with BMSCs was demonstrated to reduce the expression levels of LC-3 and Beclin-1, subsequently inhibiting autophagic activity and mitigating the damage associated with silicosis.

\section{Introduction}

Silicosis is a serious occupational disease that is characterized by the formation of early inflammatory cell nodular lesions and late silicotic nodules, ultimately leading to progressive silicosis (1). Autophagy may be present in silicosis and can affect the incidence of silicosis. Autophagy is a biological process conducted in the majority of eucaryotic cells, which is also known as 'cell cannibalism'. During autophagy, cellular structures are degraded by lysosomal activity and the cytoplasm is recycled in order to maintain precisely calibrated environments to ensure the function of cellular mechanisms. Thus, autophagy functions as a 'double-edged sword' in the fate of controlling the cells $(2,3)$. Autography is a normal process in cell growth, development and homeostasis, and aids the maintenance of a balance between the synthesis, degradation and subsequent recycling of cellular products $(3,4)$. Optimum rates of autophagy protect cells against apoptosis and necrosis; however, excessive activation of autophagy can result in type II programmed cell death (5).

A previous study indicated that bone marrow-derived mesenchymal stem cells (BMSCs) may be effective in the treatment of silicosis (6). BMSCs are a type of multipotent stem cell, derived from the bone marrow, which possess multidirectional differentiation potential (7). Furthermore, BMSCs exhibit a marked immune function during treatment, 
which can prevent the immune response-mediated rejection of allografts (8). In addition, BMSCs generate a variety of paracrine cytokines that are effective for the promotion of angiogenesis and wound repair (9).

In the present study, a rat model of silicosis was established via the administration of silicon dioxide $(\mathrm{SiO} 2)$ to the rat bronchus using bronchoalveolar lavage (BAL). Alveolar macrophages (AMs) were extracted using the BAL, and the activation of autophagy in the AMs of the silicosis model rats was evaluated using various morphological and biochemical analytical methods. Thus, the aim of the present study was to determine the expression levels of the autophagy-related proteins, microtubule-associated protein light chain 3 (LC-3) and autophagy-related gene Beclin-1 (Beclin-1), in the AMs of the silicosis model rats using morphological and biochemical detection methods, and to thereby provide an experimental basis for the further study of the pathogenesis of silicosis.

\section{Materials and methods}

Ethical approval. All procedures were performed in accordance with the Institutional Guidelines for the Care and Use of Laboratory Animals (Hebei United University, TangShan, Hebei, China), and conformed to the National Institutes of Health (NIH) Guide for the Care and Use of Laboratory Animals (NIH Publication no. 80-23, revised 1996).

BMSC culture. BMSCs were extracted from 5 male Sprague-Dawley (SD) rats. Fresh bone marrow cells were collected by flushing the medullary cavity of the rat femurs with Dulbecco's modified Eagle's medium (DMEM; Gibco Life Technologies, Grand Island, NY, USA). After filtering, the cells were centrifuged at $167.7 \mathrm{x}$ g for $5 \mathrm{~min}$. The purified cells were dispersed in cell culture flasks (Corning, Inc., Corning, NY, USA), grown in DMEM media supplemented with $10 \%$ fetal calf serum (Gibco Life Technologies), $100 \mathrm{U} / \mathrm{ml}$ penicillin and $100 \mu \mathrm{g} / \mathrm{ml}$ streptomycin (Sigma-Aldrich, St. Louis, $\mathrm{MO}$, USA), and subsequently cultured at $37^{\circ} \mathrm{C}$ with $5 \% \mathrm{CO}_{2}$. After $48 \mathrm{~h}$, the non-adherent cells were removed and fresh media was added, with the media subsequently exchanged every three days. Adhered cells were allowed to grow to $\sim 90 \%$ confluency, after which the cells were trypsinized and reseeded. Third-generation (P3) BMSCs were used for all the subsequent experiments.

Flow cytometry. P3 BMSCs were harvested via trypsinization (Gibco Life Technologies), and fixed in neutralized 2\% paraformaldehyde solution for $30 \mathrm{~min}$. Fixed cells were washed three times with phosphate-buffered saline (PBS) and incubated for 25-30 min with antibodies against the following cell surface antigens: CD29, anti-mouse monoclonal (cat. no. 102205); CD45, rabbit polyclonal (cat. no. 202205); CD54, rabbit polyclonal (cat. no. 202405); and CD106, anti-mouse monoclonal (cat. no. 200403; all 1:200; Santa Cruz Biotechnology, Inc., Dallas, TX, USA). The primary antibodies were directly conjugated with fluorescein isothiocyanate (CD29 and CD45) or phycoerythrin (CD54 and CD106). Flow cytometry was performed using fluorescence-activated cell sorting (FACS Calibur $^{\mathrm{TM}}$; BD Biosciences, Franklin Lakes, NJ, USA).
Animals and silicosis model. BMSCs were extracted from five male SD rats (age, 6-8 weeks; weight, 200-220 g). The recipient population of the BMSCs consisted of 60 female SD rats (age, 3-5 weeks; weight, 100-120 g). All animals were provided by Beijing Vital River Laboratory Animal Technology Co., Ltd. (Beijing, China), and all procedures were performed in accordance with the National Institutes of Health Guide for the Care and Use of Laboratory Animals (NIH Publication no. 80-23, revised 1996). The rat model of silicosis was induced using a single $1.0-\mathrm{ml}$ infusion of $\mathrm{SiO}_{2}$ suspension (5 g/l; Sigma-Aldrich), which was administered by non-exposed tracheal intubation. All the rats were maintained in a room with a reversed 12-h light-dark cycle at $21^{\circ} \mathrm{C}$ and $55 \%$ humidity.

Grouping and BMSC administration. The 60 female recipient rats were allocated at random into three groups, namely the control, model and BMSC treatment groups ( $\mathrm{n}=20$ per group). Control group rats received a $1.0-\mathrm{ml}$ intratracheal instillation of sterile $\mathrm{NaCl}$ solution, while the model group rats received a $1.0-\mathrm{ml}$ intratracheal instillation of sterile $\mathrm{NaCl}$ solution and $\mathrm{SiO} 2$ dust suspension $(5 \mathrm{mg} / \mathrm{ml})$. The BMSC treatment group received the same $1.0-\mathrm{ml} \mathrm{SiO}_{2}$ treatment $(5 \mathrm{~g} / \mathrm{l})$ as the model group, followed by a 1.0-ml suspension of BMSCs $(1 \times 106 / \mathrm{ml})$ in DMEM at $12 \mathrm{~h}$ after the administration of the $\mathrm{SiO}_{2}$. The rats in each group received $10 \%$ chloral hydrate $(3 \mathrm{ml} / 100 \mathrm{~g})$ prior to being sacrificed by decapitation. Five rats were sacrificed on each of days 1, 7, 14 and 28 following treatment, after which AMs were extracted using BAL.

AM extraction. AMs were acquired using BAL, following which centrifugation was conducted at $377.325 \mathrm{x}$ g for $10 \mathrm{~min}$ at $4^{\circ} \mathrm{C}$. The supernatant was discarded, and the precipitate containing the AMs and other cell types was collected. The AMs were enriched and purified using the cell adhesion method, in which the precipitate of the BAL fluid was washed 2-3 times with saline and cultivated in a high glucose medium. The cells were subsequently resuspended in DMEM with $10 \%$ fetal bovine serum, and diluted in a suspension at $1 \times 10^{6}$ cells $/ \mathrm{ml}$.

Hematoxylin and eosin (HE) staining. The AMs were placed on a glass slide, fixed with paraformaldehyde and subsequently washed with running water. The specimens were subjected to gradient alcohol dehydration and cleared with xylene, after which the cytoplasm was subsequently stained with HE. Finally, the samples were mounted with neutral gum and observed under a microscope (AE31 inverted biological microscope; Motic Incorporation Ltd., Causeway Bay, Hong Kong, China).

Immunocytochemical staining. The specimen slides were purified and enriched, soaked in water to remove formaldehyde and washed with PBS 2-3 times. The AMs were subjected to Triton lysis for 15-20 min, incubated with $3 \% \mathrm{H} 2 \mathrm{O} 2$ for 10-15 min, antigen retrieval was performed with trypsin at room temperature for $20 \mathrm{~min}$, and sealed in $5 \%$ bovine serum albumin (Wuhan Boster Biological Engineering, Wuhan, China) for $20 \mathrm{~min}$. Next, the specimens were incubated with rabbit anti-rat antibodies targeting LC-3 (PM036; 1:50; MBL International Corporation, Woburn, MA, USA) and Beclin-1 (ab195792; 1:100; Abcam, Cambridge, UK) at $4^{\circ} \mathrm{C}$ overnight. 
A

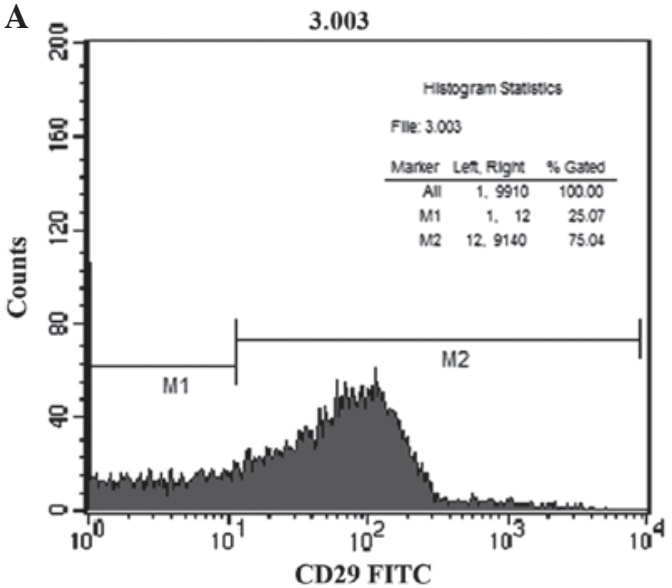

C

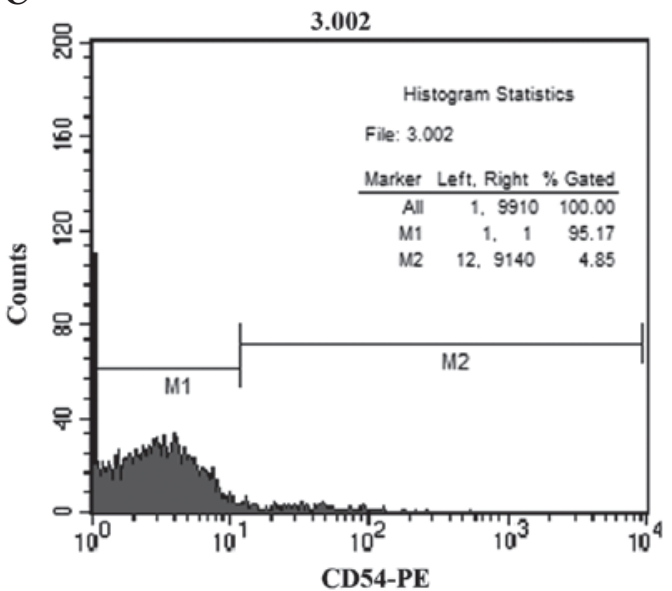

B

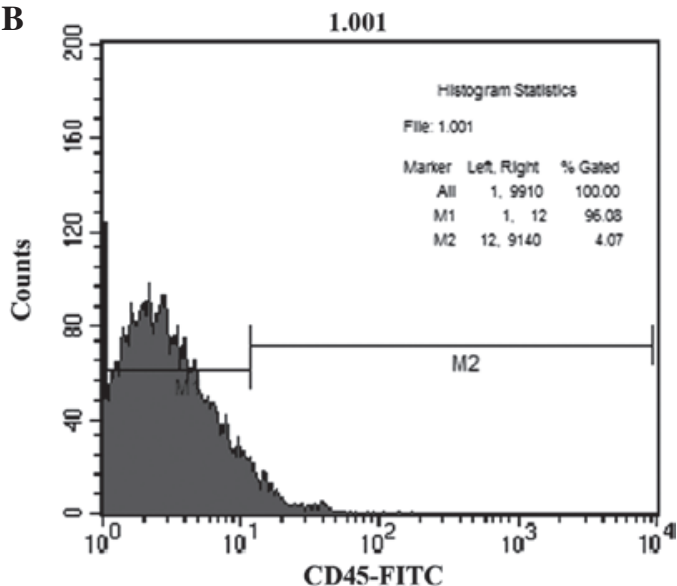

D

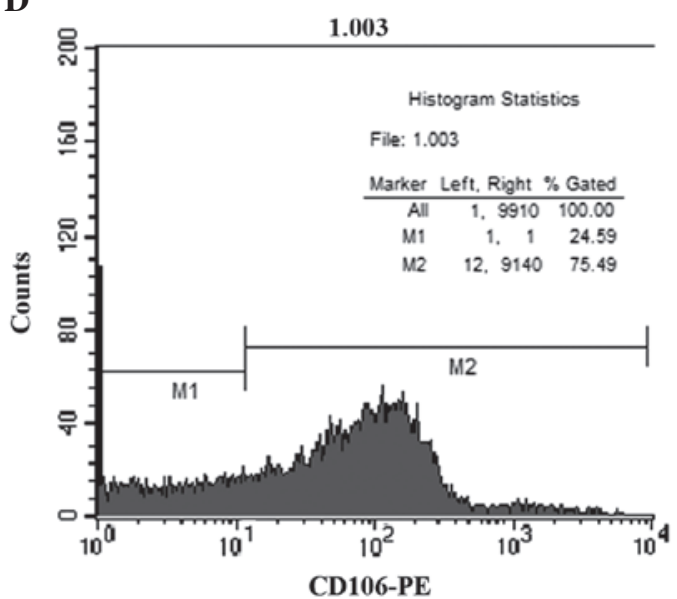

Figure 1. Expression of the bone marrow-derived mesenchymal stem cell surface markers (A) CD29, (B) CD45, (C) CD54 and (D) CD106 via flow cytometry. Positive cell rates for CD29, CD106, CD45 and CD54 were 75.04, 75.49, 4.07 and 4.85\%, respectively. FITC, fluorescein isothiocyanate; PE, phycoerythrin.

After the samples were adjusted to room temperature, a goat anti-rabbit secondary antibody (074-1506; 1:5,000; KPL, Inc., Gaithersburg, MA, USA) was added and incubated at $37^{\circ} \mathrm{C}$ for $30 \mathrm{~min}$, followed by a streptavidin-biotin-peroxidase complex, which was incubated at $37^{\circ} \mathrm{C}$ for $20 \mathrm{~min}$. Specimens were visualized using 3,3'-diaminobenzidine chromogenic solution, dehydrated using an alcohol gradient, clarified with dimethylbenzene and xylene and mounted with neutral gum. A negative control was constructed using an identical quantity of PBS instead of primary antibody, with all other protocol steps unchanged.

Western blot analysis. Following removal of the cell culture flask, the drained culture medium was washed with PBS three times. Radioimmunoprecipitation assay lysis buffer was added to cover the cell surface, and the solution was allowed to stand on ice for 10-15 min. The cells were removed from the surface using a clean and dry brush, and stored in an Eppendorf tube. Following centrifugation at $2,414.8 \times \mathrm{g}$ for $15 \mathrm{~min}$ at $4^{\circ} \mathrm{C}$, the supernatant, which contained the cellular proteins, was extracted. The total proteins were extracted and the protein concentration was determined using a bicinchoninic acid assay (Beijing Solarbio Science \& Technology Co., Ltd., Beijing, China). Samples were subjected to sodium dodecyl sulfate polyacrylamide gel electrophoresis, and the separated proteins were transferred onto polyvinylidene difluoride membranes (Roche Diagnostics GmbH, Mannheim, Germany). The immunoblots were blocked with 5\% fat-free dry milk for $1 \mathrm{~h}$ at room temperature. Following blocking, the membranes were incubated overnight at $4^{\circ} \mathrm{C}$ with polyclonal rabbit anti-LC3 (1:500) and anti-Beclin-1 (1:200) antibodies and a monoclonal mouse (074-1806; 1:5,000; KPL, Inc.) anti- $\beta$-actin primary antibody (cat. no. M1210-2; 1:500; Epigentek Group Inc., Farmingdale, NY, USA). Next, the immunoblots were subsequently incubated with horseradish peroxidase-conjugated anti-rabbit IgG and anti-mouse IgG (1:5,000; Cell Signaling Technology, Inc., Danvers, MA, USA) for $2 \mathrm{~h}$ at room temperature. The immunoblot on the membrane was visualized following development using an enhanced chemiluminescence detection system (ChemiDoc XRS; Bio-Rad Laboratories, Inc., Hercules, CA, USA), and the densitometric signals were quantified using an imaging program (Digital Medical Image Analysis System 6.0; Motic). Immunoreactive bands for all the proteins examined were normalized against $\beta$-actin. Western blot results were analyzed using the National Institutes of Health Image software, version 1.41 (Bethesda, MD, USA).

Statistical analysis. All experiments were repeated three times and similar results were obtained. Statistical analysis was performed using SPSS software, version 16.0 (SPSS, Inc., 

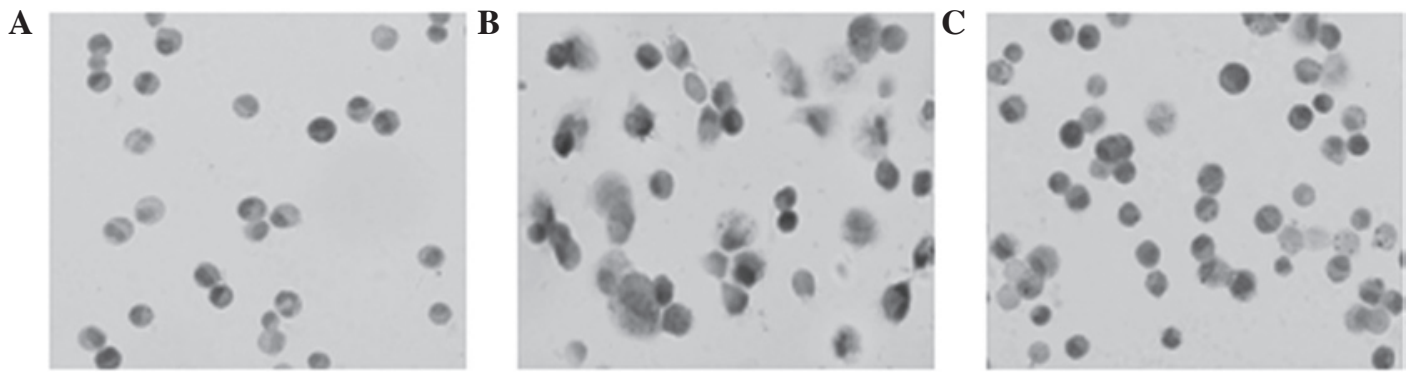

Figure 2. Morphology of (A) control, (B) model and (C) bone marrow-derived mesenchymal stem cell-treated alveolar macrophages on day 14, visualized using hematoxylin and eosin staining (magnification $\mathrm{x} 400$ ).

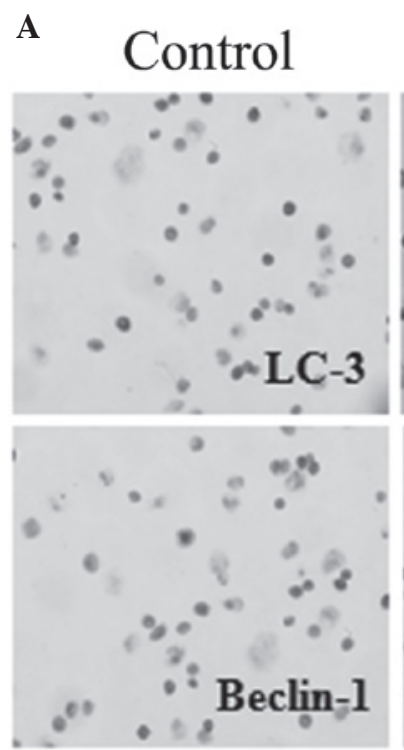

B Silica
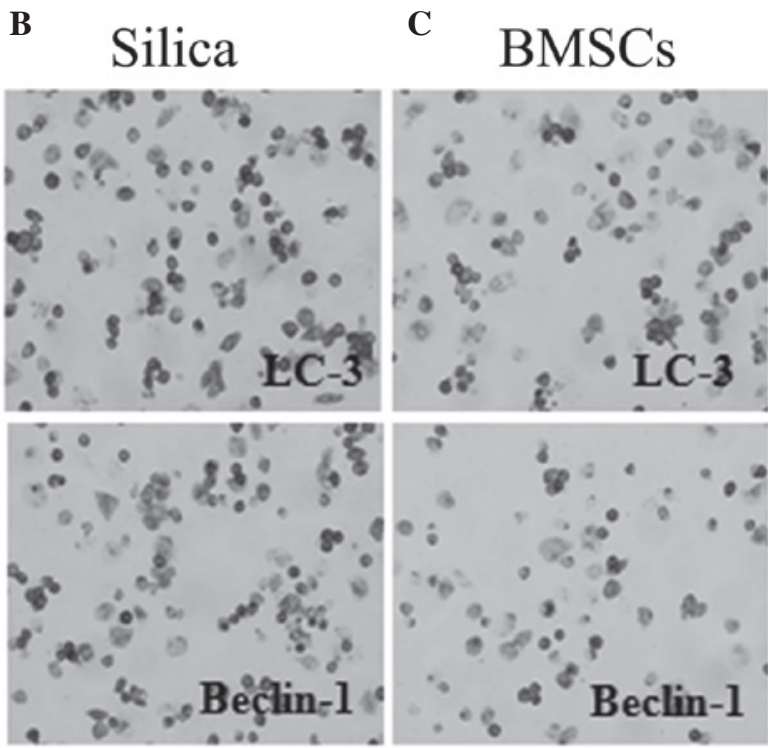

Figure 3. Immunohistochemical staining results of microtubule-associated protein light chain 3 and autophagy-related Beclin-1 protein in the (A) control, (B) model and (C) bone marrow-derived mesenchymal stem cell-treated groups (magnification, $\mathrm{x} 400$ ). A darker coloration indicates positive cell expression.

Chicago, IL, USA). Data are expressed as the mean \pm standard error of the mean. The statistical significance of the experimental results was determined using one-way analysis of variance, where $\mathrm{P}<0.05$ was considered to indicate a statistically significant difference.

\section{Results}

BMSC growth rate and cell surface marker detection.P3 BMSCs were identified using flow cytometry to detect the expression of a number of cell surface antigens. Tshe results of these experiments revealed that the cell positivity rates for CD29 and CD106 antibodies were 75.04 and $75.49 \%$, respectively. However, the cell positivity rates for the expression of CD45 and CD54 antibodies were 4.07 and $4.85 \%$, respectively (Fig. 1), indicating that the BMSCs were positive for the expression of CD29 and CD106, but negative for the expression of CD45 and CD54. Thus, the cells were confirmed as BMSCs, and the cells used in the subsequent experiments were P3 BMSCs.

Morphology of the AMs. Results from the HE staining revealed that the control group AMs were almost all round or oval, and the nuclei were located in the center or inclined to one side of the cells. In addition, the cytoplasm was unchanged, with no evident engulfed $\mathrm{SiO}_{2}$ dust particles. When compared with the control group, the cell volume of the AMs in the silicosis model group increased (Fig. 2). Furthermore, the majority of the cells presented with irregular, basophilic nuclei, and were colored in the center or side of the cell cytoplasm.

Location of LC-3 and Beclin-1 protein. Cytoplasmic expression of the autophagy-associated proteins, LC-3 and Beclin-1, appeared as brown particles in the AMs. The results indicated that the control group cells were highly homogeneous, with a clear structure and limited expression of LC-3 and Beclin-1 (Fig. 3). When compared with the control group, the AMs in the model group exhibited a significantly increased cell size, the shape and size was not uneven, and the cell profile was not clear, with brown or reddish-brown coloration, indicating a marked immune response. The BMSC treatment group presented with significantly reduced numbers of LC-3- and Beclin-1-positive cells when compared with the model group, indicating attenuated immune reactivity.

BMSC treatment inhibits the expression of LC-3 and Beclin-1 in silicosis model AMs. In the control group, limited basal expression levels of LC-3 and Beclin-1 protein were detected, with negligible variation over time. However, the expression 


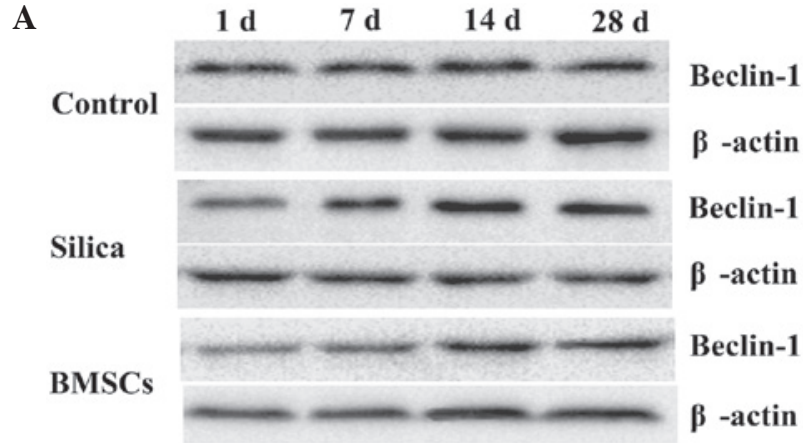

B

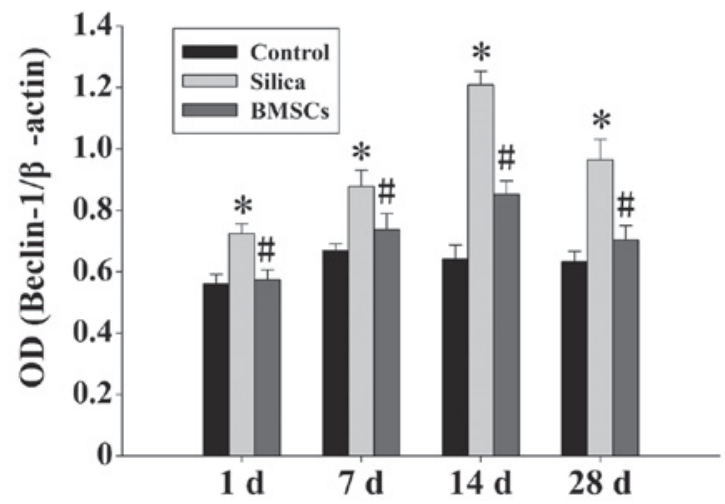

Figure 4. Western blot analysis showing Beclin-1 protein expression. (A) Western blot bands of Beclin-1 and (B) densitometry analysis of the Beclin-1 band corresponding to $\beta$-actin. ODs of the respective protein bands were analyzed using Image $\mathbf{J}$ software. Bars represent the mean \pm standard error of the mean ( $\mathrm{n}=5$ per group). ${ }^{\text {" }} \mathrm{P}<0.05$, vs. control group; ${ }^{\#} \mathrm{P}<0.05$, vs. model group. Beclin-1, autophagy-related gene Beclin-1; BMSC, bone marrow-derived mesenchymal stem cell; OD, optical density.

levels of LC-3 and Beclin-1 protein in the model group AMs were significantly increased at each time point when compared with the control group AMs (Figs. 4 and 5; $\mathrm{P}<0.05$ ). The expression levels of LC-3 and Beclin-1 were shown to be dynamic, increasing at day 1 and peaking at day 14, while decreasing after day 28 . However, the expression levels of LC-3 and Beclin-1 after day 28 in the silicosis model AMs remained elevated compared with those in the control group AMs at the corresponding period. When compared with the AMs in the model group, LC-3 and Beclin-1 protein expression levels were significantly decreased in the BMSC treatment group AMs at each time point (Figs. 4 and $5 ; \mathrm{P}<0.05$ ); however, the expression trend was similar, with peak expression observed on day 14 . The expression levels of LC-3 were presented as two bands in different shades, representing LC-3I and LC-3II (Fig. 5).

\section{Discussion}

Silicosis is a occupational disease with a complex pathogenesis. AM injury is a crucial factor in the development of silicosis (10). A previous study (11) extracted AMs using a BAL in situ technique, and then performed in vitro transfection of the cells with $\mathrm{SiO} 2$ in order to establish a model of $\mathrm{SiO} 2$ damage for subsequent analysis (12). The disadvantage of this approach is that the cells have been removed from the native environment of the organism, in which coexisting cells exert a variety of effects and interactions. A previous study by the present research group indicated that autophagy is activated
A

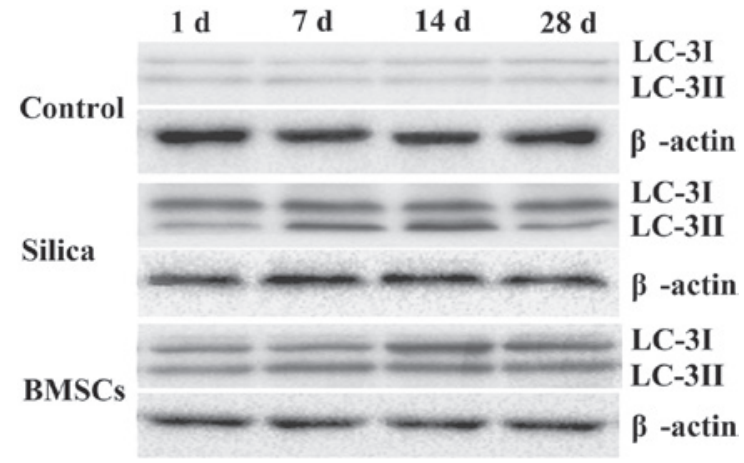

B

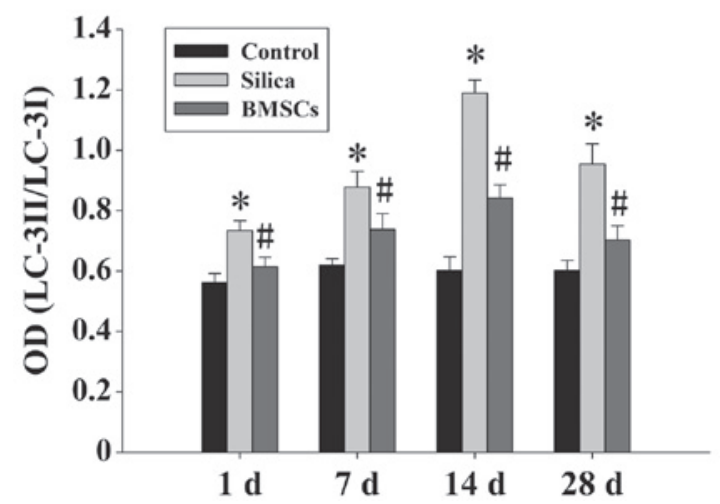

Figure 5. Western blot analysis showing LC-3 protein expression. (A) Western blot bands of LC-3 and (B) densitometry analysis of the LC-3 band corresponding to $\beta$-actin. Bars represent the mean \pm standard error of the mean ( $\mathrm{n}=5$ per group). ${ }^{*} \mathrm{P}<0.05$, vs. control group; ${ }^{*} \mathrm{P}<0.05$, vs. model group. LC-3, microtubule-associated protein light chain 3; BMSC, bone marrow-derived mesenchymal stem cell; OD, optical density.

in the lung tissue of rats with silicosis. The current study was conducted on the basis of the aforementioned study using an in vivo rat model of silicosis, induced by the $\mathrm{SiO} 2$ exposure method. However, in the present study, the AMs remained in the living environment in the organism following exposure to $\mathrm{SiO} 2$. Using BAL in situ techniques, the AMs were removed from the organism and follow-up experiments were conducted in vitro. This approach can effectively avoid the in vitro transfection of dust and interference caused by external factors, such as dust particles in the air.

Autophagy is a key process in cellular energy metabolism and a mechanism of self-renewal. The cellular process aids the maintenance of body homeostasis via a degradation/recycling system, which supports biosynthesis, metabolism, growth and aging (13). Autophagy was initially considered to be a type of programmed cell death; however, subsequent studies (14) demonstrated that autophagy and apoptosis are different processes. Apoptosis results in cell death without exception, while autophagy may aid cell survival, since the autophagic degradation and recycling of the internal cellular structure may help cells adapt to external stimuli. Therefore, autophagy serves a dual function in the control of cell fate (15). However, the in vivo experiments of the present study confirmed that autophagy is able to promote the pathogenesis of silicosis. These results indicate the control of autophagy as a novel treatment approach, and signify autophagy as a target for candidate drugs for the treatment of silicosis.

Recently, BMSCs have been increasingly recognized as applicable to the treatment of lung injury. A number of studies 
have indicated that the exogenous administration of BMSCs may protect against a variety of pulmonary diseases, including acute lung injury $(16,17)$ and chronic lung disease (18). BMSCs exhibit a marked capacity for self-renewal, multidirectional differentiation (19), perception of the position of damage signals, repair and regeneration (20), immune function and the ability to prevent the immune rejection of allografts (21). Furthermore, paracrine function, angiogenesis promotion and the ability to repair associated damage are key factors in the treatment of silicosis and necessary mechanisms (11). In addition, BMSC transplantation has been demonstrated to inhibit the expression of autophagy-associated proteins in hippocampal cells in a rat model of traumatic brain injury (22). For these experiments provide the basic theory to ensure the feasibility of the experiment.

The present study observed autophagic activation on day 1 in the AMs following the successful modeling of silicosis in rats. Furthermore, the expression levels of LC-3 and Beclin-1 continued to increase, peaking at day 14 and decreasing after day 28. These results confirm that AM autophagic activity occurs during the formation and development of silicosis. Pathological changes were significantly alleviated in the BMSC treatment group when compared with the model group. However, the expression trend of LC-3 and Beclin-1 remained unchanged, while the overall degree of expression declined. These results indicate that the transplantation of BMSCs may be used to reduce autophagic activity, thereby effectively inhibiting the progress of silicosis caused by AM damage and promoting fibrotic lung recovery. Therefore, BMSC transplantation may be clinically applicable as a therapeutic intervention for the treatment of silicosis.

In summary, a model of silicosis was established via the administration of free $\mathrm{SiO} 2$ dust in situ in order to maintain a response to body interactions. The results indicate that AMs may be crucially involved in the initiation and progression of the lung tissue inflammation and fibrosis associated with silicosis. The present study employed a silicosis model manufactured in vivo, while the experiments were conducted in vitro. The occurrence and participation of autophagic activity in the development and progression of silicosis was confirmed in the AMs of the rats. Furthermore, BMSC transplantation therapy was demonstrated to effectively reduce the expression of autophagy-associated proteins, and promote the recovery of AMs. Thus, the present study provides a novel theoretical and experimental basis for the treatment of silicosis.

\section{Acknowledgements}

This study was supported by grants from the Science and Technology Support Key Funding Project of Hebei Province (no. 09276191D) and Hebei Province Occupational Disease Prevention and Control Research (no. 13277709D).

\section{References}

1. Lee E, Lee EJ, Kim H, Jang A, Koh E, Uh ST, Kim Y, Park SW and Park CS: Overexpression of apolipoprotein A1 in the lung abrogates fibrosis in experimental silicosis. PLoS One 8: e55827, 2013.
2. Debnath J, Baehrecke EH and Kroemer G: Does autophagy contribute to cell death? Autophagy 1: 66-74, 2005.

3. Shintani T and Klionsky DJ: Autophagy in health and disease: A double-edged sword. Science 306: 990-995, 2004.

4. Mizushima N: Autophagy: Process and function. Genes Dev 21: 2861-2873, 2007.

5. Hale AN, Ledbetter DJ, Gawriluk TR and Rucker EB III: Autophagy: Regulation and role in development. Autophagy 9: 951-972, 2013.

6. Derubeis AR and Cancedda R: Bone marrow stromal cells (BMSCs) in bone engineering: Limitations and recent advances. Ann Biomed Eng 32: 160-165, 2004.

7. Tohma Y, Dohi Y, Ohgushi H, Tadokoro M, Akahane M and Tanaka Y: Osteogenic activity of bone marrow-derived mesenchymal stem cells (BMSCs) seeded on irradiated allogenic bone. J Tissue Eng Regen Med 6: 96-102, 2012.

8. Zhang YG, Guo X, Xu P, Kang LL and Li J: Bone mesenchymal stem cells transplanted into rabbit intervertebral discs can increase proteoglycans. Clin Orthop Relat Res: 219-226, 2005.

9. Zheng JF and Liang LJ: Intra-portal transplantation of bone marrow stromal cells ameliorates liver fibrosis in mice. Hepatobiliary Pancreat Dis Int 7: 264-270, 2008.

10. Yao SQ, He QC, Yuan JX, Chen J, Chen G, Lu Y,Bai YP,Zhang CM, Yuan Y and Xu YJ: Role of Fas/FasL pathway-mediated alveolar macrophages releasing inflammatory cytokines in human silicosis. Biomed Environ Sci 26: 930-933, 2013.

11. Zhao MM, Cui JZ, Cui Y, Li R, Tian YX, Song SX, Zhang J and Gao JL: Therapeutic effect of exogenous bone marrow-derived mesenchymal stem cell transplantation on silicosis via paracrine mechanisms in rats. Mol Med Rep 8: 741-746, 2013.

12. Ferreira TPT, de Arantes ACS, do Nascimento CVMF, Olsen PC, Trentin PG, Rocco PR, Hogaboam CM, Puri RK, Martins MA and Silva PM: IL-13 immunotoxin accelerates resolution of lung pathological changes triggered by silica particles in mice. J Immunol 191: 5220-5229, 2013

13. Johansen $\mathrm{T}$ and Lamark T: Selective autophagy mediated by autophagic adapter proteins. Autophagy 7: 279-296, 2011.

14. Chaabane W, User SD, El-Gazzah M, Jaksik R, Sajjadi E, Rzeszowska-Wolny $\mathrm{J}$ and Los MJ: Autophagy, apoptosis, mitoptosis and necrosis: Interdependence between those pathways and effects on cancer. Arch Immunol Ther Exp (Warsz) 61: 43-58, 2013.

15. Shen S, Kepp O, Michaud M, Martins I, Minoux H, Métivier D, Maiuri MC, Kroemer RT and Kroemer G: Association and dissociation of autophagy, apoptosis and necrosis by systematic chemical study. Oncogene 30: 4544-4556, 2011.

16. Islam MN, Das SR, Emin MT, Wei M, Sun L, Westphalen K, Rowlands DJ, Quadri SK, Bhattacharya S and Bhattacharya J: Mitochondrial transfer from bone-marrow-derived stromal cells to pulmonary alveoli protects against acute lung injury. Nat Med 18: 759-765, 2012.

17. Luan Y, Zhang X, Kong F, Cheng GH, Qi TG and Zhang ZH: Mesenchymal stem cell prevention of vascular remodeling in high flow-induced pulmonary hypertension through a paracrine mechanism. Int Immunopharmacol 14: 432-437, 2012.

18. Aslam M, Baveja R, Liang OD, Fernandez-Gonzalez A, Lee C, Mitsialis SA and Kourembanas S: Bone marrow stromal cells attenuate lung injury in a murine model of neonatal chronic lung disease. Am J Respir Crit Care Med 180: 1122-1130, 2009.

19. Soleimani M, Abbasnia E, Fathi M, Sahraei H, Fathi Y and Kaka G: The effects of low-level laser irradiation on differentiation and proliferation of human bone marrow mesenchymal stem cells into neurons and osteoblasts - an in vitro study. Lasers Med Sci 27: 423-430, 2012.

20. Le Blanc K and Ringdén O: Immunomodulation by mesenchymal stem cells and clinical experience. J Intern Med 262: $509-525,2007$.

21. Grove JE, Lutzko C, Priller J, Henegariu O, Theise ND, Kohn DB and Krause DS: Marrow-derived cells as vehicles for delivery of gene therapy to pulmonary epithelium. Am J Respir Cell Mol Biol 27: 645-651, 2002.

22. Sun L, Gao J, Zhao M, Jing X, Cui Y, Xu X, Wang K, Zhang W and Cui J: The effects of BMSCs transplantation on autophagy by CX43 in the hippocampus following traumatic brain injury in rats. Neurol Sci 35: 677-682, 2014. 